\title{
Charge Displacements in Interfacial Layers Containing Reaction Centers
}

\author{
P. Brzezinski ${ }^{1}$, A. Messinger ${ }^{2,5}$, Y. Blatt ${ }^{3}$, A. Gopher ${ }^{4}$, D. Kleinfeld ${ }^{5}$ \\ ${ }^{1}$ Department of Biochemistry and Biophysics, Göteborg University and Chalmers University of Technology (Medicinaregatan 9C), P.O. Box \\ 462, SE-405 30 Göteborg, Sweden \\ ${ }^{2}$ Salk Institute for Biological Studies, 10010 North Torrey Pines Road, La Jolla, CA 92037-1099, USA \\ ${ }^{3}$ Bio Dar Ltd., Yavne Technology Park, Building 10, P.O. Box 344, Yavne 81103, Israel \\ ${ }^{4}$ Institute of Pharmacology and Toxicology, Sheba Medical Center, Tel Hashomer, Israel \\ ${ }^{5}$ Department of Physics, University of California, 9500 Gilman Drive, La Jolla, CA 92093-0319, USA
}

Received: 1 July 1997/Revised: 23 June 1998

\begin{abstract}
Reaction centers from the photosynthetic bacterium Rhodobacter sphaeroides were oriented in phospholipid interfacial layers adsorbed to a Teflon film separating two electrolyte-filled compartments of a Teflon cell. Light-induced voltage changes were measured as a function of time across electrodes immersed in the cell compartments. The experimental system is characterized both experimentally and theoretically to relate the measured signals to the light-induced displacement currents in the reaction centers. Mathematical relations between the measured signals and the distances and geometries of the charge-transfer reactions are derived. At $\mathrm{pH}$ 8.0 the reaction centers were found to be oriented with $\sim 60 \%$ of the population oriented with the donor facing the aqueous phase. The density of the reaction centers in the layer was $\sim 10^{11} \mathrm{~cm}^{-2}$, which is close to that found in the native system. Reconstitution of the secondary quinone, $Q_{B}$, in $90 \%$ of the RCs was achieved with an $\sim 100$-fold excess of ubiquinone in the vesicle preparation.
\end{abstract}

Key words: Bacterial photosynthesis - Electrogenic event - Charge transfer - Electron transfer - Monolayer

\section{Introduction}

Reaction centers $(R C s)$ from photosynthetic bacteria convert light energy into electrochemical energy. The

Correspondence to: P. Brzezinski
$R C$ s are membrane-bound protein complexes that accommodate a number of cofactors associated with the conversion process. In Rhodobacter sphaeroides RCs there are four bacteriochlorophylls, two bacteriopheophytins, one nonheme iron $\left(\mathrm{Fe}^{2+}\right)$ and two ubiquinones [reviewed by Feher et al., 1989].

The energy conversion is initiated by a light-induced charge separation in which a photon excites a bacteriochlorophyll dimer $(P)$. Following the excitation an electron is transferred sequentially from $P$, via an intermediate phoephytin, to the primary and secondary quinone acceptors $Q_{A}$ and $Q_{B}$, stabilizing the charge-separated state for progressively longer times. The oxidized donor is rereduced by a water-soluble cytochrome, cyt. $c_{2}$. After a second charge separation, $Q_{B}$ becomes doubly reduced and binds two protons from the cytoplasmic side of the membrane [reviewed in Crofts \& Wraight, 1983]. The dihydroquinone dissociates from the $R C$ and is replaced by an unprotonated quinone from the quinone pool in the membrane (Diner et al., 1984; McPherson, Okamura \& Feher, 1990). In intact bacterial photosynthetic membranes the reoxidation of $Q_{B} H_{2}$ is coupled to the release of protons to the periplasmic side of the membrane.

Since the membrane-bound $R C$ is an insulating medium, the motion of charges with components perpendicular to the membrane surface is associated with displacement currents and voltage changes, collectively defined as electrogenic events. The electrogenic events associated with some reactions that involve charge transfer have been previously studied monitoring the carotenoid band shifts in chromatophores (Witt, 1971; Jackson \& Dutton, 1973) and by direct electrical measure- 




Fig. 1. Electron-transfer reactions in the RCs of $R$. sphaeroides. Following the absorption of a photon, an electron is transferred from the excited donor $\mathrm{P}^{*}$, via an intermediate pheophytin acceptor (omitted for simplicity), to $Q_{A}$ and then to $Q_{B}$. Transfer rates are given for room temperature and rounded to the nearest power of 10. Charge recombination from $Q_{B}^{-}$to the oxidized donor, $P^{+}$, proceeds via the intermediate state $\mathrm{P}^{+} \mathrm{Q}_{A}^{-} \mathrm{Q}_{B}$.

ments of $R C \mathrm{~s}$ in artificially constructed lipid layers (Schönfeld, Montal \& Feher, 1979; Packham et al., 1980, 1982; Tiede, Mueller \& Dutton, 1982; Trissl, 1983; Apell, Snozzi \& Bachofen, 1983; Gopher et al., 1985; Feher, Arno \& Okamura, 1988; Höök \& Brzezinski, 1994; Brzezinski et al., 1997) and colloidal films (Drachev et al., 1976; Dracheva et al., 1988).

In the present study we measured the voltages and currents evoked upon illumination of $R C \mathrm{~s}$ oriented in a phospholipid interfacial layer adsorbed to a Teflon film, a technique that was pioneered to study rhodopsin (Trissl, Darszon \& Montal, 1977) and bacteriorhodopsin (Trissl \& Montal, 1977; Hong \& Montal, 1979). These direct electrical measurements have the advantage of being sensitive not only to electron transfer but also to processes not generally associated with spectral changes, such as structural changes involving charge motion (Brzezinski et al., 1992) and proton transfer in the $R C \mathrm{~s}$ (Brzezinski et al., 1991, 1997). The Teflon-supported interfacial layer provides a simple, robust (compared to planar bilayers) and well-defined experimental system. In this paper we describe the electrogenic measurement technique and characterize the system of $R C \mathrm{~s}$ in a phospholipid interfacial layer using the electrogenic events associated with the charge separation and recombination reactions between the primary donor $P$ and the quinones $Q_{A}$ and $Q_{B}$ (see Fig. 1). Characterization of the system makes it possible to quantitatively analyze various electrogenic events such as electron transfer, proton uptake and conformational changes.

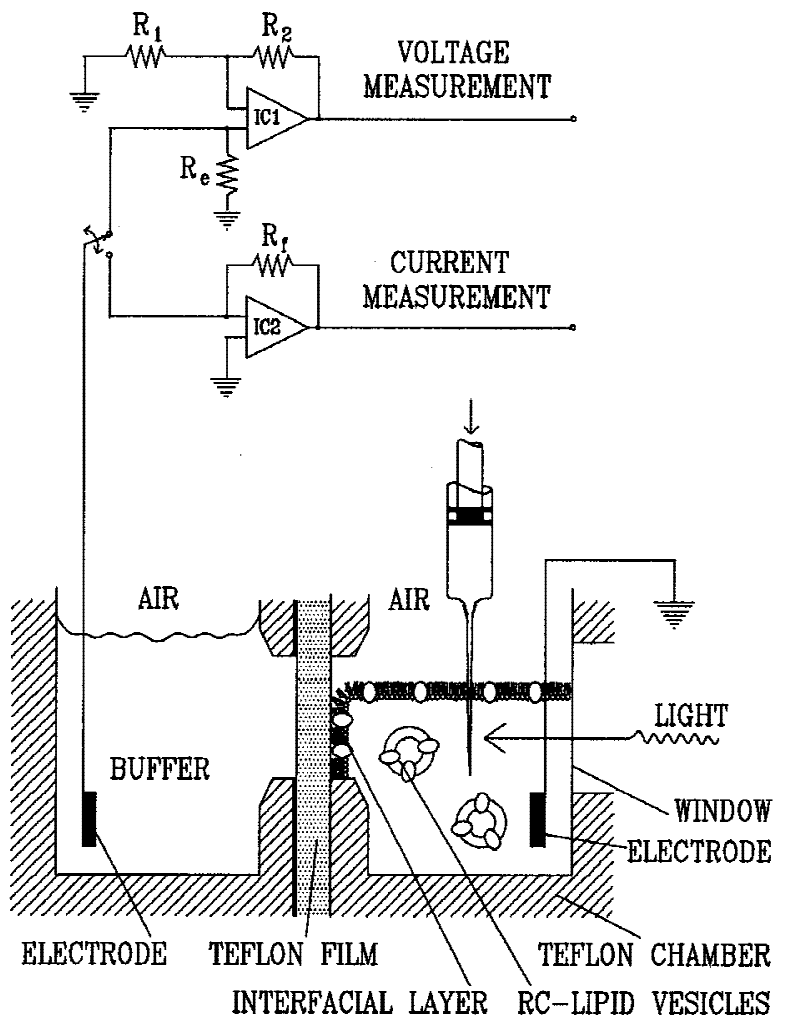

Fig. 2. Schematic representation of the experimental setup. The chamber consists of two compartments, separated by a Teflon film $(6 \mu \mathrm{m}$ thick). The interfacial layer was formed by raising the level of the vesicle solution in the right-hand side compartment until the exposed Teflon film was completely covered. The interfacial layer was illuminated through a glass window. One of the electrodes was connected to the input of the operational amplifier (IC1 or IC2) and the other electrode was grounded. The electrodes were shielded from the incident light. The values of the resistors are: $R_{1}=4 \mathrm{k} \Omega, R_{2}=40 \mathrm{k} \Omega, R_{e}=$ $10^{8}-10^{12} \Omega, R_{f}=10^{9} \Omega$. The figure is not to scale.

\section{Materials and Methods}

\section{REACTANTS}

RCs from Rhodobacter sphaeroides R-26 were purified as described (Feher \& Okamura, 1978) with lauryl dimethylamine N-oxide (LDAO, Onyx Chem, NJ) as a solubilizing detergent. $R C$ s with only one quinone, i.e., $Q_{A}$, were prepared using the method of Okamura, Isaacson \& Feher (1975). Cytochrome $c$ (horse heart type VI, Sigma, MO) was more than $95 \%$ reduced with hydrogen gas, using platinum black (Aldrich, WI) as a catalyst (Rosen \& Pecht, 1976). Soybean lecithin (Sigma, Type IIS, MO) was washed as described (Kagawa \& Racker, 1971).

\section{The EXPERIMENTAL SETUP}

The experimental setup is shown in Fig. 2. The chamber was made of black Teflon (Fluorocarbon, CA). It consists of two compartments separated by a $6.3 \mu \mathrm{m}$ thick Teflon film (Saunders, CA). The area of the Teflon film was $0.3 \mathrm{~cm}^{2}$. The measured signals were found to be 
more reproducible if the Teflon film was coated with a hydrophobic agent on the side to receive the layer. We used $6 \mu l$ of a $1 \%$ hexadecane solution in heptane. Excess of the solution was evaporated by an intense flow of air.

\section{Preparation of the Lipid Vesicles}

Lipid vesicles containing $R C$ s were prepared as described earlier (Gopher et al., 1985) by suspending $10 \mathrm{mg}$ of lipids in $1 \mathrm{ml}$ of buffer composed (in $\mathrm{mM}$ ) of: $10 \mathrm{KCl}, 2.5$ sodium citrate, 2.5 PIPES, 2.5 HEPPS, 2.5 CHES and 2.5 CAPS at $\mathrm{pH} 8.0^{1}$. To prepare $R C$ s with two quinones per $R C$, phospholipid and ubiquinone ( $\mathrm{UQ}_{10}$, Sigma, $\mathrm{MO}$ ) were dried together from a hexane solution prior to dispersion in the above solution. A quinone-to- $R C$ ratio of 100:1 gave at least $90 \%$ of $R C$ s with an active $Q_{B}$. The above solution was supplemented with 5 nmole of $R C \mathrm{~s}(\leqslant 50 \mu \mathrm{l})$ in $0.025 \%$ LDAO and sonicated for $\sim 10 \mathrm{~min}$ at $22 \pm 2{ }^{\circ} \mathrm{C}$ until the solution was optically clear as evidenced from the $650 \mathrm{~nm}$ absorbance.

\section{PREPARATION OF THE RC-LIPID INTERFACIAL LAYER}

The vesicle solution was diluted to a final concentration of $1 \mathrm{mg}$ lipid/ $\mathrm{ml}$ in the buffer above, supplemented with $10 \mathrm{mM} \mathrm{CaCl}_{2}$ and $200 \mathrm{~mm}$ $\mathrm{KCl}$. The solution was added to one of the compartments of the cell up to a level below that of the Teflon film. The liquid level in the compartment was raised slowly, allowing the monolayer formed at the surface to adhere to the Teflon (Fig. 2). The opposing cell compartment was filled with an aqueous salt solution $(\sim 200 \mathrm{mM} \mathrm{KCl})$.

\section{LigHT SOURCES}

Pulsed illumination of the $R C$-lipid interfacial layer was provided by a flashlamp-pumped dye laser (Phase R, Model DL 1200V, NH) using the dye Rhodamine (Phase R, NH), which had a maximal output energy at $590 \mathrm{~nm}$. The pulse had an energy of $\sim 100 \mathrm{~mJ}$ and a width of $\sim 0.4$ $\mu \mathrm{sec}$. Continuous illumination was provided by a tungsten lamp (maximum $1 \mathrm{~W} \mathrm{~cm}^{-2}$ ) filtered through $2 \mathrm{~cm}$ of water. The duration of the illumination was varied using a shutter (Uniblitz, Vincent, NY) with a time constant of $\sim 1 \mathrm{msec}$.

\section{ELECTRICAl MEASUREMENTS}

Measurements were carried out in a way similar to that described (Schönfeld et al., 1979; Feher et al., 1988). The light-induced voltage or current was detected using Calomel (Ingold Electrodes, MA) or $\mathrm{Ag} / \mathrm{AgCl}$ electrodes, shielded from actinic light. Voltage changes were measured using an operational amplifier (IC1 in Fig. 2, Burr-Brown OP128) connected as a voltage follower with a shunt resistance, $R_{e}$ (Fig. 2). The shunt resistor prevented current, leaking from the amplifier, from charging the Teflon film.

Currents were measured with an operational amplifier (IC2 in Fig. 2, Burr-Brown OP121) connected as a current to voltage converter with a feedback resistor of $10^{9} \Omega\left(R_{f}\right.$ in Fig. 2$)$. The output signals were filtered electronically and averaged using a transient recorder

\footnotetext{
${ }^{1}$ The buffer composition made it possible to use the same buffer conditions in the $\mathrm{pH}$ range $4-11$.
}

(Nicolet, model 1180, Madison, WI). Capacitance was measured with a capacitance bridge (Electro Scientific Industries, model 7073, Portland, OR).

\section{ANALYSIS}

Signals were averaged and fitted with exponential functions using a nonlinear least square procedure (PeakFit, Jandel, San Rafael, CA). The standard deviation of the results obtained on the same interfacial layer was considerably smaller (5\% for kinetics and $6 \%$ for amplitudes) than that obtained from different interfacial layers prepared under similar conditions (9\% for kinetics and $20 \%$ for amplitudes). The error reported hereafter is the standard deviation of the mean; typically 8-10 interfacial layers were prepared. The charge recombination reactions $P^{+} Q_{A}^{-} \rightarrow P Q_{A}$ and $P^{+} Q_{A} Q_{B}^{-} \rightarrow P Q_{A} Q_{B}$ (Fig. 1) were identified by comparing the time constants of the exponentially decaying currents to those obtained from optical measurements on $R C$ s in detergent solutions (Okamura et al., 1982) and in vesicle solutions.

\section{ABbREVIATIONS AND Symbols}

CAPS, 3-(cyclohexylamino) propanesulfonic acid; CHES, 2(cyclohexylaminoethanesulfonic acid); HEPPS, 4-(2-hydroxyethyl)-1piperazinepropanesulphonic acid; PIPES, 1,4-piperazinediethanesulphonic acid; RC, reaction center; P, bacteriochlorophyll dimer; $Q_{A}, Q_{B}$, ubiquinones.

$D_{T}$, $D_{L}$ thickness, $C_{T}, C_{L}$ capacitance, $A_{T}, A_{L}$ area, $\varepsilon_{T}, \varepsilon_{L}$ dielectric constant of the Teflon film and of the lipid layer, respectively; $\tau_{l}$, $\tau_{2}$, electric relaxation time constants; $v_{j k}(t), i_{j k}(t)$, voltage across and current through the interfacial layer associated with a charge transfer from a donor $j$ to an acceptor $k ; V_{j k}(t), I_{j k}(t)$, measured voltage and current associated with the same charge transfer; $q_{j k}$, charge transferred per $R C$ from a donor $j$ to an acceptor $k ; \vec{d}_{j k}$, distance traveled by charges in a reaction in which $j$ is the donor and $k$ is the acceptor; $\varepsilon_{j k}$, dielectric constant between $j$ and $k$; $\Theta$, effective orientation of the RCs in the interfacial layer; $N_{S}, N_{T}$, number of $R C$ s in the $S$ population ( $P$ faces the aqueous solution) and $T$ population ( $P$ faces the Teflon film); $\sigma$, population density of the $R C \mathrm{~s}$ in the interfacial layer; $\alpha_{j k}$, dielectric coefficient (Läuger, 1991), which defines the fractional, dielectrically weighted distance traveled by the charge.

\section{Theoretical Models}

\section{The Equivalent Electrical Circuit}

To establish the electrical characteristics of the experimental system (Fig. 3A) we modeled it with an equivalent electrical circuit (Fig. $3 B$ ). The light-induced $R C$ generated current is represented by the current generator output, $i(t)$. Because the $R C$ is a transmembrane protein, the current generator is in parallel with the resistance $R_{L}$ and capacitance $C_{L}$ of the lipid layer. The capacitance of the Teflon film, $C_{T}$, is in series with $R_{L}$ and $C_{L}$. The measured current, $I(t)$, is the ac-coupled current through the Teflon film when the external resistance $R_{e}$ is zero. The measured voltage, $V(t)$, is the potential produced across the external resistance $R_{e}$ by the ac-coupled through the Teflon film. The series resistance of the 



Fig. 3. (A) Schematic representation of the structure of the $R C$-lipid interfacial layer adsorbed on the Teflon film. $A$ one-quinone $R C$ from the $S$ population (donor facing the aqueous solution) in the charge separated state $P^{+} Q_{A}^{-}$is shown in the interfacial layer. $\vec{d}_{A P}$ is the distance vector from $Q_{A}$ to $P$. The unit vector perpendicular to the interfacial layer, $\hat{n}$, is shown. The interfacial layer is assumed to have an average thickness of $\sim 20 \AA$. However, it is likely that in the vicinity of each $R C$, which is $75 \AA$ long, a bilayer is maintained. $(B)$ Equivalent electrical circuit of the experimental system. The portion within the dashed line models the $R C$-lipid interfacial layer adsorbed on the Teflon film. The light-induced current produced by the $R C \mathrm{~s}$ is modeled by the output of the current generator, $i(t) . R_{L}$ is the resistance and $C_{L}$ the capacitance of the interfacial layer. $I(t)$ is the capacitively-coupled current through the Teflon film capacitor $\left(C_{T} \cong 95 \mathrm{pF}\right)$. The measured voltage, $V(t)$, is the potential across the external resistance, $R_{e}$, due to the current $I(t) . R_{e}$ was adjusted to make the charging time $\tau_{2}=R_{e} C_{T}$ of the Teflon film capacitor long compared to the electrogenic event. For current measurements $R_{e}$ is assumed to be $0 \Omega$, and the measured current, $I(t)$, is assumed to flow to a virtual ground. In reality, the finite resistance of the solution and amplifier will limit the time resolution of the current measurement. However, the response time of the experimental setup was $<<1 \mu \mathrm{sec}$, and therefore this resistance is not relevant for the present studies. The equivalent circuit treats the resistance of the Teflon film as infinite and that of the buffer solution and the electrodes as zero.

buffer in each compartment was not included in the equivalent circuit because its resistance is so small (30 $\Omega$ ) that the voltage drop across it is negligible compared to the voltage across the interfacial layer.

An alternate equivalent circuit has been discussed in the literature in which the signal generator is ac-coupled to the output through a "chemical capacitance" (Hong, 1980; Trissl, 1981; Okajima \& Hong, 1986). The equivalent circuit in Fig. $3 B$ models the measured signal without the need of a chemical capacitance because the $R C$-generated current, $i(t)$, is already ac-coupled to the output through the Teflon-film capacitor (Fahr, Läuger \& Bamberg, 1981; Rayfield, 1982).

\section{The Capacitance of the Teflon Film and of the INTERFACIAL LAYER}

The measured capacitance of the Teflon film was $95 \mathrm{pF}$. This value is consistent with the calculated capacitance $(90 \mathrm{pF})$ for a parallel plate capacitor with an area of 0.30 $\mathrm{cm}^{2}$ and a thickness of $6.3 \mu \mathrm{m}$, using a dielectric constant of 2.1 relative to vacuum for Teflon (Westman, 1956). The capacitance per unit area of an $\sim 40 \AA$ thick soybean lecithin bilayer is $0.75 \mu \mathrm{F} / \mathrm{cm}^{2}$ (Nelson et al., 1980 ) and is essentially unchanged when RCs are incorporated into the bilayer (Gopher et al., 1985). Assuming that the interfacial layer is half as thick as the bilayer, the capacitance per unit area of the interfacial layer should be $1.5 \mu \mathrm{F} / \mathrm{cm}^{2}$. The interfacial layer in the sample cell then has $C_{L}=0.45 \mu \mathrm{F}$, which is much larger than $C_{T}$. Consequently, the capacitance of the interfacial layer, which is in series with $C_{T}$, could not be measured electrically. Note that we here assume that the average monolayer thickness is $\sim 20 \AA$. However, it is likely in the immediate vicinity of each $R C$ a bilayer is formed, which covers the hydrophobic part of the $R C$ s (see also legend to Fig. 3).

\section{The Electrical Time Constants}

The measured voltage and current, $V(t)$ and $I(t)$, are determined by the electrogenic events occurring in the $R C$ (as described by $i(t)$ ) and the electrical relaxation processes of the electrical circuit (i.e., charge motion outside of the $R C$ ). To reliably determine the kinetics of the electrogenic reactions, their time constants must be small compared to the time constants of the electrical circuit.

The electrical time constants were derived from an analysis of the equivalent electrical circuit (Fig. $3 B$ and Appendix A). For $C_{L} \gg C_{T}$, the two electrical time constants in the voltage mode are approximately:

$\tau_{1} \cong R_{L} C_{L} \quad \tau_{2} \cong R_{e} C_{T}$

where $\tau_{l}$ is the time constant for the voltage across the interfacial layer, $v(t)$, to discharge due to leakage of ions through the interfacial layer. The time constant $\tau_{2}$ is associated with the charging of the capacitor $C_{T}$. Initially, $C_{T}$ is uncharged and $V(t)=v(t)$. As $C_{T}$ charges, the voltage across it increases with a concomitant decrease in $V(t)$ with time constant $\tau_{2}$.

When making current measurements, $R_{e}=0$ and the amplifier input is virtually grounded (Figs. 2 and $3 B$ ). Consequently, $v(t)$ is equal to the voltage across $C_{T}$ alone and there is only a single electrical time constant which, for $C_{L} \gg C_{T}$, is $\tau_{l}$.

\section{The Relation Between the Measured Signals and} a Charge Transfer Spanning the Interfacial Layer

From the equivalent electrical circuit (Fig. $3 B$ ), the measured voltage, $V(t)$, equals the voltage across the inter- 
facial layer, $v(t)$, minus the voltage across $C_{T}$. For $t<<$ $\tau_{2}, C_{T}$ is essentially uncharged and the measured voltage, $V(t)$, equals $v(t)$. For current measurements, $R_{e}=0$, hence $v(t)$ is equal to the voltage across $C_{T}$. Therefore, the measured voltage, $V(t)$, and the measured current, $I(t)$, are related by:

$V(t) \cong v(t) \cong \frac{1}{C_{T}} \int_{0}^{t} I\left(t^{\prime}\right) d t^{\prime}$

where the time integral of $I(t)$ gives the charge on $C_{T}$ at time $t$.

For $C_{L} \gg C_{T}$ and $t<<\tau_{1}$, the $R C$-generated current, $i(t)$, flows almost exclusively through $C_{L}$ (i.e., $i(t) \cong$ $\left.i_{2}(t)\right)$. Therefore, the voltage generated across the interfacial layer,

$v(t) \cong \frac{1}{C_{L}} \int_{o}^{t} i\left(t^{\prime}\right) d t^{\prime}$

is equal to the charge transferred across the interfacial layer up to time $t$ (i.e., the time integral of the displacement current) divided by $C_{L}$. Equations 2 and 3 show that the measured current, $I(t)$, is given by (Fahr et al., 1981; see also Läuger, 1991),

$I(t) \cong \frac{C_{T}}{C_{L}} i(t)$.

The Relation Between the Measured Signals and a Charge Transfer within the Interfacial Layer

The $R C$-generated current in the equivalent electrical circuit (Fig. $3 B$ ) flows through the entire interfacial layer, as represented by $C_{L}$ and $R_{L}$. But the transfer of charge within the RC from a donor $j$ to an acceptor $k$ generates a current, $i_{j k}(t)$, which flows through only part of the interfacial layer. For this case, the voltage, $v_{j k}(t)$, generated across the interfacial layer by $i_{j k}(t)$ is found by replacing $C_{L}$ in Eq. 3 with the capacitance between sites $j$ and $k$ (Fahr et al., 1981). This capacitance is equal to $C_{L}$ times the dielectrically weighted ratio of the thickness of the interfacial layer to the distance between sites $j$ and $k$. If all the donor and acceptor sites lie within a slab of dielectric constant $\varepsilon_{j k}$ which is interior and parallel to the planar faces of the interfacial layer then, for $C_{L} \gg C_{T}$ and $t<<\tau_{l}$,

$v_{j k}(t)=\frac{1}{C_{L}} \alpha_{j k} \int_{o}^{t} i_{j k}\left(t^{\prime}\right) d t^{\prime}$ with $\alpha_{j k} \cong \frac{\varepsilon_{L}}{\varepsilon_{j k}} \frac{\vec{d}_{j k} \cdot \hat{n}}{D_{L}}$

where $\varepsilon_{L}$ is the average dielectric constant of the interfacial layer, $D_{L}$ is the average thickness of the layer around the $R C \mathrm{~s}, \vec{d}_{j k}$ the distance vector from $j$ to $k$, and $\hat{n}$ is a unit vector perpendicular to the layer (Fig. $3 A$ ). The constant $\alpha_{j k}$ is a dielectric coefficient (Läuger, 1991), which defines the fractional, dielectrically weighted distance traveled by the charge.

Note that the voltage generated is proportional to the induced dipole moment (i.e., charge transferred times the distance vector between $j$ and $k$ ) and inversely proportional to the dielectric constant between $j$ and $k$. Equation 5 has appeared previously without an exact proof (Läuger et al., 1981) and is derived in Appendix B. Because of the simplicity of the plane parallel model of the interfacial layer, Eq. 5 should be taken only as a semiquantitative approximation.

For charge transfer within the $R C, C_{T}$ in Eq. 2 should, in principle, be replaced by the capacitance of the Teflon film and the portion of the interfacial layer not spanned by the charges (Fahr et al., 1981). For $C_{L} \gg$ $C_{T}$, this capacitance is negligibly different from $C_{T}$ so Eq. 2 still applies. The relative size of the measured and $R C$-generated current for a dipole-forming charge transfer within the $R C$ is obtained by combining Eqs. 2 and 5:

$I_{j k}(t)=\frac{C_{T}}{C_{L}} \alpha_{j k} i_{j k}(t)$

We have assumed in this section that the electrical relaxation processes can be neglected (i.e., $t<<\tau_{1}$ and $\left.\tau_{2}\right)$. Under these conditions, the measured signals $V(t)$ and $I(t)$ have the same kinetics as the $R C$-generated current. In Appendix A, the measured signals are related to an exponentially decaying $R C$-generated current, taking into account the effects of the electrical relaxation processes.

At time, $t_{\max }$, long compared to the time constant of the electrogenic process $\left(\tau_{0}\right.$ in Appendix A) but short compared to the electrical time constants $\tau_{1}$ and $\tau_{2}$, one obtains from Eqs. 2 and 6, the maximum measured voltage change,

$\Delta V_{j k}(t) \cong \Delta v_{j k}(t)=\frac{N q_{j k}}{C_{L}} \alpha_{j k}$

and the maximum integral of the measured current,

$\int_{o}^{t_{\max }} I_{j k}(t) d t \cong \frac{C_{T}}{C_{L}} N q_{j k} \alpha_{j k}$

The EfFective Orientation of the RCs IN THE INTERFACIAL LAYER

The $R C \mathrm{~s}$ in the interfacial layer are composed of two populations. In one population the donors face the aqueous solution (the $S$ population, see Fig. $3 A$ ) and in the 
other the donors face the Teflon film (the $T$ population). A charge transfer in one population produces a signal with the opposite sign as the signal produced by the same charge transfer in the other population. The observed net signal originates from processes occurring in both populations simultaneously. The experimental observation of a net signal indicates that the $S$ and $T$ populations do not contribute equally.

The asymmetry in the contributions from the $S$ and $T$ populations may be due to differences in the electrical properties of the two populations, differences in the number of $R C \mathrm{~s}$ in the two populations, or differences in the reactions that occur in the two populations. We define the effective orientation,

$\Theta \cong \frac{w_{S} N_{S}-w_{T} N_{T}}{w_{S} N_{S}+w_{T} N_{T}}$

where $N_{S}$ and $N_{T}$ are the number of $R C$ s in the $S$ and $T$ populations, respectively, and $w_{S}$ and $w_{T}$ are weighting factors that account for other possible differences in the properties of the $S$ and $T$ populations, respectively. For example, $R C$ s in the $S$ population may be exposed to a different environment and/or may be electrostatically shielded differently than $R C \mathrm{~s}$ in the $T$ population. For simplicity we assume that the effective orientation observed is due solely to a difference in the number of RCs in each population, i.e., $N_{S} \neq N_{T}$ and $w_{S}=w_{T}$.

To measure the effective orientation we compare the signals from both the $S$ and $T$ populations to that from only the $T$ population. Both signals are observed with one-quinone $R C \mathrm{~s}$. When the interfacial layer is illuminated a transient current due to the charge separation $P Q_{A} \rightarrow P^{+} Q_{A}^{-}$is observed. When the illumination ceases, a transient current of opposite sign due to the charge recombination $P^{+} Q_{A}^{-} \rightarrow P Q_{A}$ is observed.

Using Eq. 8, the time integral of the measured current, $I_{A P}(t)$, associated with charge recombination ${ }^{2}$ is related to the net charge transferred in the $R C \mathrm{~s}$ during charge recombination (i.e., the time integral of the $R C$ generated current):

$\int_{o}^{t_{\max }} I_{A P}(t) d t \cong \frac{C_{T}}{C_{L}}\left(N_{s}-N_{T}\right) q_{A P}\left|\alpha_{A P}\right|$

where $\alpha_{\mathrm{AP}}$ is the dielectric coefficient between $Q_{A}$ and $P$. Because the charges are displaced in opposite directions in the two populations, the net charge transferred is equal to the difference in the number of $R C \mathrm{~s}$ in the two popu-

\footnotetext{
${ }^{2}$ The integrals of the charge separation and recombination currents are the same since charge is conserved. We discuss the charge recombination here as this process is slower which makes the integral easier to determine precisely.
}

lations times $q_{A P}$, the charge transferred between $Q_{A}$ and $P$ in each $R C$ (one electron charge).

To observe only the $T$ population, cytochrome $c^{2+}$ was added to the aqueous solution on the interfacial layer side. Cytochrome $c$ binds to the $R C$ surface near the donor. Cytochrome $c$ was found not to penetrate the interfacial layer so that the donor was only accessible in the $S$ population. Thus, following the first illumination cytochrome reduced the oxidized donor $P^{+}$in the $\mathrm{S}$ population only, forming the inactive $P Q_{A}^{-}$state. Consequently, upon cessation of the illumination only the $T$ population recombined to the initial $P Q_{A}$ state, whereas the $S$ population remained in the photochemically inactive state $P Q_{A}^{-}$on the time scale of the measurements. Following a second illumination, charge separation and recombination took place only in the $T$ population. Consequently, the time integral of the measured current, $I_{A P}(t)$, due to charge recombination in the presence of cytochrome $c^{2+}$ is given by,

$\int_{0}^{t_{\max }} I_{A P}^{c}(t) d t \cong \frac{C_{T}}{C_{L}}\left(-N_{T}\right) q_{A P}$

which is proportional to the size of the T population.

Equations 9 (with $w_{S}=w_{T}$ ), 10 and 11 combine to give the effective orientation:

$$
\begin{aligned}
\Theta & =\frac{N_{S}-N_{T}}{N_{S}+N_{T}}=\frac{N_{S}-N_{T}}{\left(N_{S}-N_{T}\right)+2 N_{T}} \\
& =\frac{\int_{0}^{t_{\max }} I_{A P}(t) d t}{\int_{O}^{t_{\max }} I_{A P}(t) d t-2 \int_{0}^{t_{\max }} I_{A P}^{c}(t) d t} .
\end{aligned}
$$

The currents, $I_{A P}(t)$ and $I_{A P}^{c}(t)$, associated with charge recombination $P^{+} Q_{A}^{-} \rightarrow P Q_{A}$ have an exponential time dependence and decay with the same time constant ${ }^{3}, \tau_{A P}$. Consequently, $\int_{O}^{t_{\max }} I_{A P}(t) d t=I_{A P}(0) \tau_{A P}$ and $\int_{O}^{t_{\max }} I_{A P}^{c}(t) d t=$ $I_{A P}^{c}(0) \tau_{A P}$ and the above ratio of integrals can be expressed in terms of a ratio of currents at $t=0$ :

$$
\Theta=\frac{I_{A P}(0)}{I_{A P}(0)-2 I_{A P}^{c}(0)}
$$

Note that the effective orientation, $\Theta$, is determined solely from observed quantities and does not explicitly depend on $C_{L}, C_{T}, \varepsilon_{L}, \varepsilon_{T}, \vec{d}_{A P}$ or $q_{A P}$.

\footnotetext{
${ }^{3}$ It is assumed that the charge-recombination time constant $P^{+} Q_{A}^{-} \rightarrow$ $P Q_{A}$ is the same in both the $T$ and $S$ populations. This assumption is based on results from studies which show that this time constant is essentially insensitive to the reaction-center environment (see e.g., Schönfeld et al. 1980), as well as on the results in Fig. 7, which show that the rate of charge recombination for the $S$ and $T$ populations (Fig. $7 A$ ) is the same as that for the $T$ population alone (Fig. $7 B$ ).
} 


\section{The Density of RCs IN the InTerfacial LAyeR}

The $R C$ density in the interfacial layer was determined from the charge recombination current. The number of $R C \mathrm{~s}$ in the layer was obtained by dividing the net charge transferred in the layer (calculated from the time integral of the current) by the charge transferred in each $R C$ (one elementary charge) and the effective orientation, $\Theta$, (Eq. 9). Using Eq. 10, one obtains for the density:

$\sigma=\frac{N_{S}+N_{T}}{A_{L}}=\frac{N_{S}-N_{T}}{\Theta A_{L}} \cong \frac{C_{L}}{C_{T}} \frac{1}{\Theta A_{L}\left|\alpha_{A P}\right|} \frac{\int_{o}^{t_{\max }} I_{A P}(t) d t}{q_{A P}}$

where $A_{L}$ is the area of the interfacial layer.

\section{Results and Discussion}

\section{BASIC PhOTORESPONSE}

The voltage changes associated with charge separation and recombination were studied in $R C$ s with and without the secondary quinone, $Q_{B}$ (Fig. $4 A$ and $B$ ). When the $R C$ s were excited with actinic light, an increase in voltage associated with charge separation was observed. With continuous illumination, under saturating light conditions, the rise time was limited by the opening time of the shutter $(1 \mathrm{msec})$ (Fig. 4A). Upon cessation of illumination, a voltage decay associated with charge recombination was observed. The decay time constant was 100 msec in the absence of exogenous quinone, consistent with the $P^{+} Q_{A}^{-} \rightarrow P Q_{A}$ charge recombination. In the presence of exogenous quinone (100 quinones per $R C$ ) the decay time constant was $1 \mathrm{sec}$, consistent with the $P^{+} Q_{A} Q_{B}^{-} \rightarrow P Q_{A} Q_{B}$ charge recombination. The amplitude of the light-induced signal increased with time for 1 $\mathrm{hr}$ after the level of the vesicle solution was raised to cover the Teflon film (a similar observation was reported by Drachev et al., 1976). The signal growth could be due to an increase in the density or effective orientation of the $R C \mathrm{~s}$ in the interfacial layer. To obtain a stable signal and maximize the signal-to-noise ratio all measurements were performed at least one hour after the formation of the interfacial layer.

\section{The Electrical Time Constants}

Illumination of the interfacial layer produced a voltage (Fig. 4) associated with charge separation. Under continuous illumination, the RCs remained in the charge separated $P^{+} Q_{A}^{-}$state but the measured voltage decayed biphasically, with time constants, $\tau_{1}$ and $\tau_{2}$, due to the relaxation processes of the electrical circuit. The amplitude of the phase with the smaller time constant predomi-
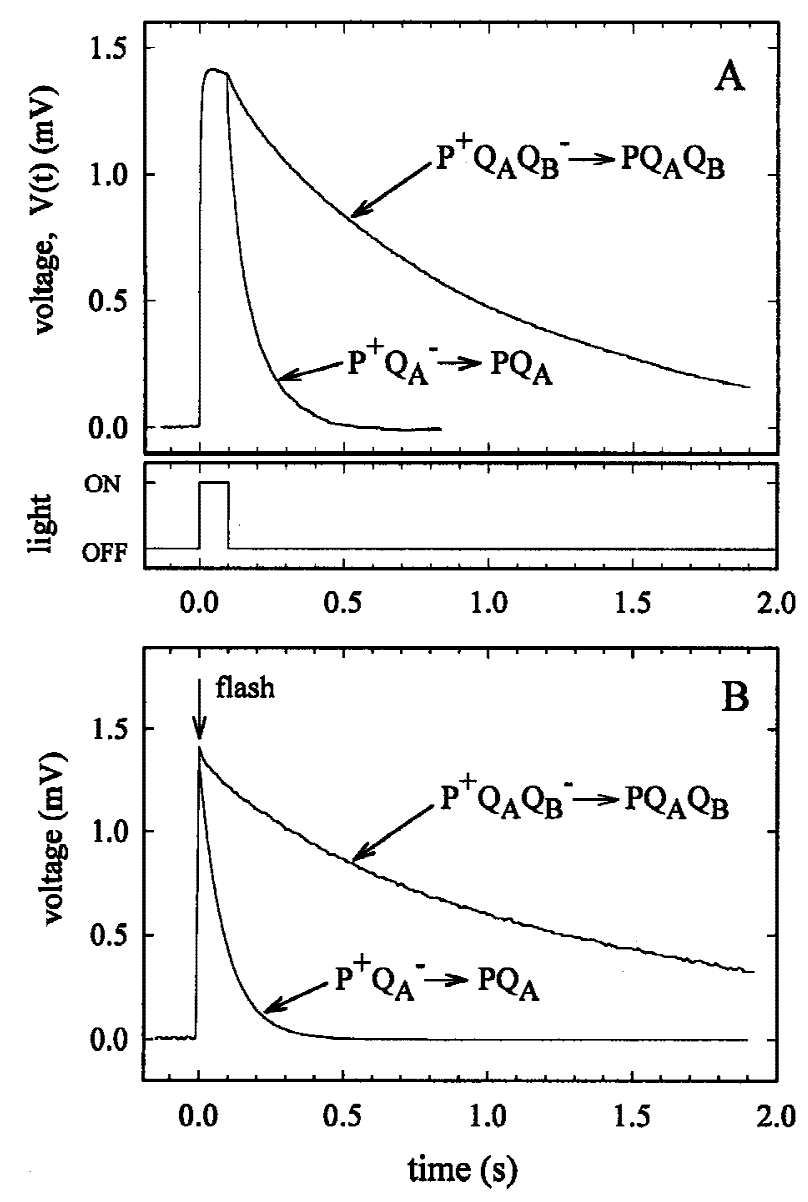

Fig. 4. Voltage changes, $V(t)$, in response to steady state $(A)$ and pulsed $(B)$ illumination of $R C$-lipid interfacial layers with and without the secondary quinone, $Q_{B}$. The initial light-induced voltage increase is due to the charge separation $P Q_{A} \rightarrow P^{+} Q_{A}^{-}$. In $(A)$ the rate of increase is limited by the opening time of the shutter $(\sim 1 \mathrm{msec})$. The traces have been scaled so that the voltage increase is equal. The electron transfer $P^{+} Q_{A}^{-} Q_{B} \rightarrow P^{+} Q_{A} Q_{B}^{-}$was not detected because the transfer occurs in a direction perpendicular to $\hat{n}$. The voltage decay following illumination has a time constant of $\sim 100 \mathrm{msec}$ in the interfacial layer containing $R C \mathrm{~s}$ that lack $Q_{B}$ and of $\sim 1 \mathrm{sec}$ in the layer containing $R C \mathrm{~s}$ with $Q_{B}(\sim 100$ $U Q_{10} / R C$ added during vesicle preparation). Reaction conditions: 0.5 $\mu \mathrm{M} R C, 0.2 \mathrm{M} \mathrm{KCl}, 10 \mathrm{~mm} \mathrm{CaCl}_{2}, 2.5 \mathrm{~mm}$ sodium citrate, $2.5 \mathrm{~mm}$ PIPES, $2.5 \mathrm{~mm}$ HEPPS, $2.5 \mathrm{~mm}$ CHES and $2.5 \mathrm{~mm}$ CAPS at $\mathrm{pH} 8.0 ; T$ $=22^{\circ} \mathrm{C}$.

nated, making the decay effectively monophasic (Appen$\operatorname{dix}$ A).

The smaller time constant, as determined from the kinetics of the voltage decay under continuous illumination, is shown as a function of $R_{e}$ in Fig. 5. For $R_{e}<<$ $10^{11} \Omega$, the smaller time constant was $\tau_{2}$, which is proportional to $R_{e}$. In this region, the charging of the Teflon film capacitor was the dominant electrical relaxation process. The constant of proportionality between $\tau_{2}$ and $R_{e}$ is the Teflon-film capacitance which was determined from the slope to be $142 \pm 16 \mathrm{pF}$. The value agrees with 


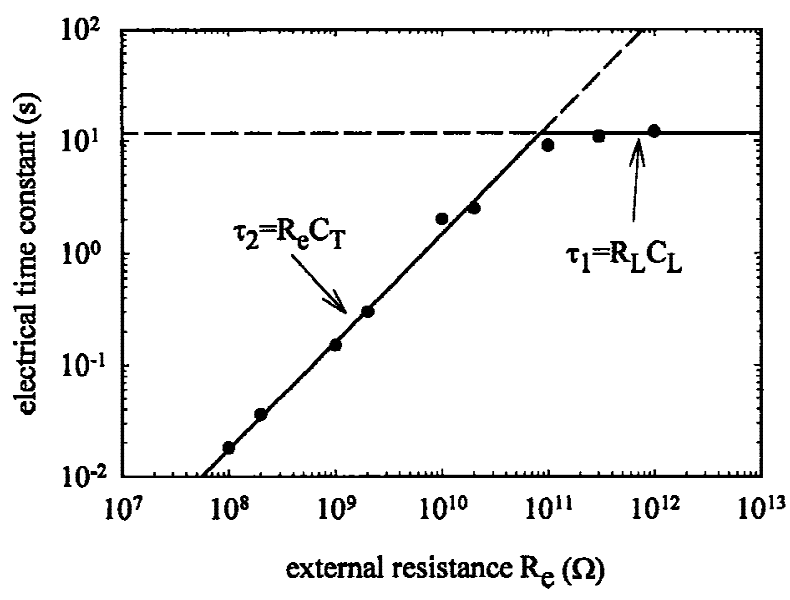

Fig. 5. The observed electrical time constant of the system as a function of the external resistance $R_{e}$ (see Fig. 3). The time constant was determined from the voltage relaxation during continuous illumination of the interfacial layer (see Appendix A). For $R_{e}<<10^{11} \Omega$, the observed electrical time constant is $\tau_{2}$, which is proportional to $R_{e}$ with a proportionality constant of $C_{T}=142 \mathrm{pF}$. For $R_{e} \gg 10^{11} \Omega$, the observed electrical time constant is $\tau_{l}(11.3 \mathrm{sec})$, which is independent of $R_{e}$. Reaction conditions same as Fig. 4.

the $130 \mathrm{pF}$ predicted from the sum of the calculated value of $C_{T}$ (see above) and $40 \mathrm{pF}$ of cable capacitance connected in parallel. As expected, a smaller slope was observed when $C_{T}$ was decreased by using a thicker Teflon film.

For $R_{e} \gg 10^{11} \Omega$, the smaller electrical circuit time constant was,

$\tau_{l}=R_{L} C_{L}=11.3 \pm 1.0 \mathrm{sec}$

which is independent of $R_{e}$. In this region, the leakage of ions through the interfacial layer was the dominant electrical relaxation process. The interfacial layer resistance was predicted to be $R_{L}=25 \pm 2 \mathrm{M} \Omega$ from the quotient of $\tau_{1}$ and the estimated (see Theoretical Models) value of $C_{L} \cong 0.45 \mu \mathrm{F}$. The corresponding interfacial layer resistance per unit area is $83 \pm 7 \mathrm{M} \Omega \mathrm{cm}^{-2}$.

To prove that the time constant of the voltage decay at large $R_{e}$ was a function of $R_{L}$ we used ionophores, which lower the resistance of lipid layers by facilitating ion transport (Läuger, 1972). The ionophores valinomycin, gramicidin, tetraphenylborate, and tetraphenylarsonium all caused a reduction of $\tau_{l}$, which proves the dependence of this time constant on $R_{L}$. We modeled $R_{L}$ as an inherent resistance (determined in the absence of ionophores) in parallel with an ionophore-induced leakage resistance. For instance, $1 \mu \mathrm{M}$ valinomycin produced a leakage resistance of $1.1 \pm 0.1 \mathrm{M} \Omega$. The leakage resistance decreased by an order of magnitude for every order of magnitude increase in the valinomycin concentration.

Most likely, around each $R C$, a lipid bilayer is main-

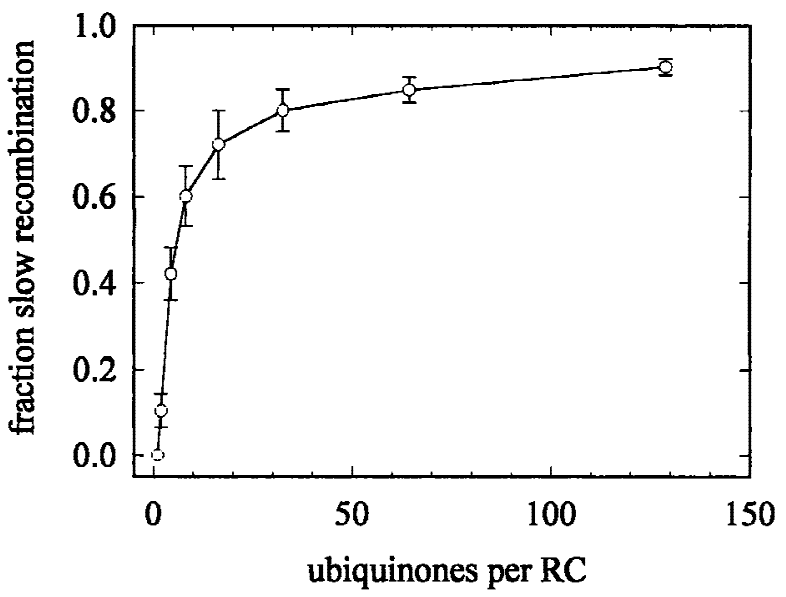

Fig. 6. Fraction of the total voltage decay (see Fig. 4) that proceeds with the slow $(\sim 1 \mathrm{sec})$ time constant associated with charge recombination from $Q_{B}$ as a function of the ubiquinone-to- $R C$ ratio. The quinone-to- $R C$ ratio is defined as the number of quinones per $R C$ added to one-quinone $R C$ s during vesicle preparation. Reaction conditions same as in Fig. 4.

tained. Consequently, a small aqueous compartment is expected to be associated with each $R C$ on the side facing the Teflon film. The effect of the ionophore would thus be to transport ions between this compartment and the aqueous phase.

\section{The Fraction of RCs Containing a Functional SECONDARY QuinONE}

The fraction of $R C$ s containing a functional secondary quinone was determined from the kinetics of the voltage changes associated with the recombination reactions following illumination of the $R C$ s (Okamura et al., 1982). The observed voltage change was decomposed into a fast $(\sim 100 \mathrm{msec})$ and slow $(\sim 1 \mathrm{sec})$ component. The fast component was ascribed to recombination in the fraction of $R C$ s lacking $Q_{B}$ and the slower component to recombination in the fraction of $R C \mathrm{~s}$ with a bound $Q_{B}$. The fraction of $R C$ s containing a functional $Q_{B}$ was determined from the relative contribution of the slow component to the total voltage change ${ }^{4}$.

Figure 6 shows the fraction of slow recombination as a function of the amount of ubiquinone (per $R C$ ) added to one quinone $R C$ s during vesicle preparation. To obtain an active $Q_{B}$ in $\sim 90 \%$ of $R C$ s in the interfacial layer required a ubiquinone $/ R C$ ratio of $\geqslant 100$ in contrast

\footnotetext{
${ }^{4}$ The voltage changes associated with the charge recombination from $P^{+} Q_{A} Q_{B}^{-}$and $P^{+} Q_{A}^{-} Q_{B}$ are equal. This was shown by observing the same voltage changes before and after addition of $200 \mu \mathrm{M}$ Terbutryn which inhibits electron transfer from $Q_{A}^{-}$to $Q_{B}$ (Wraight, 1981).
} 

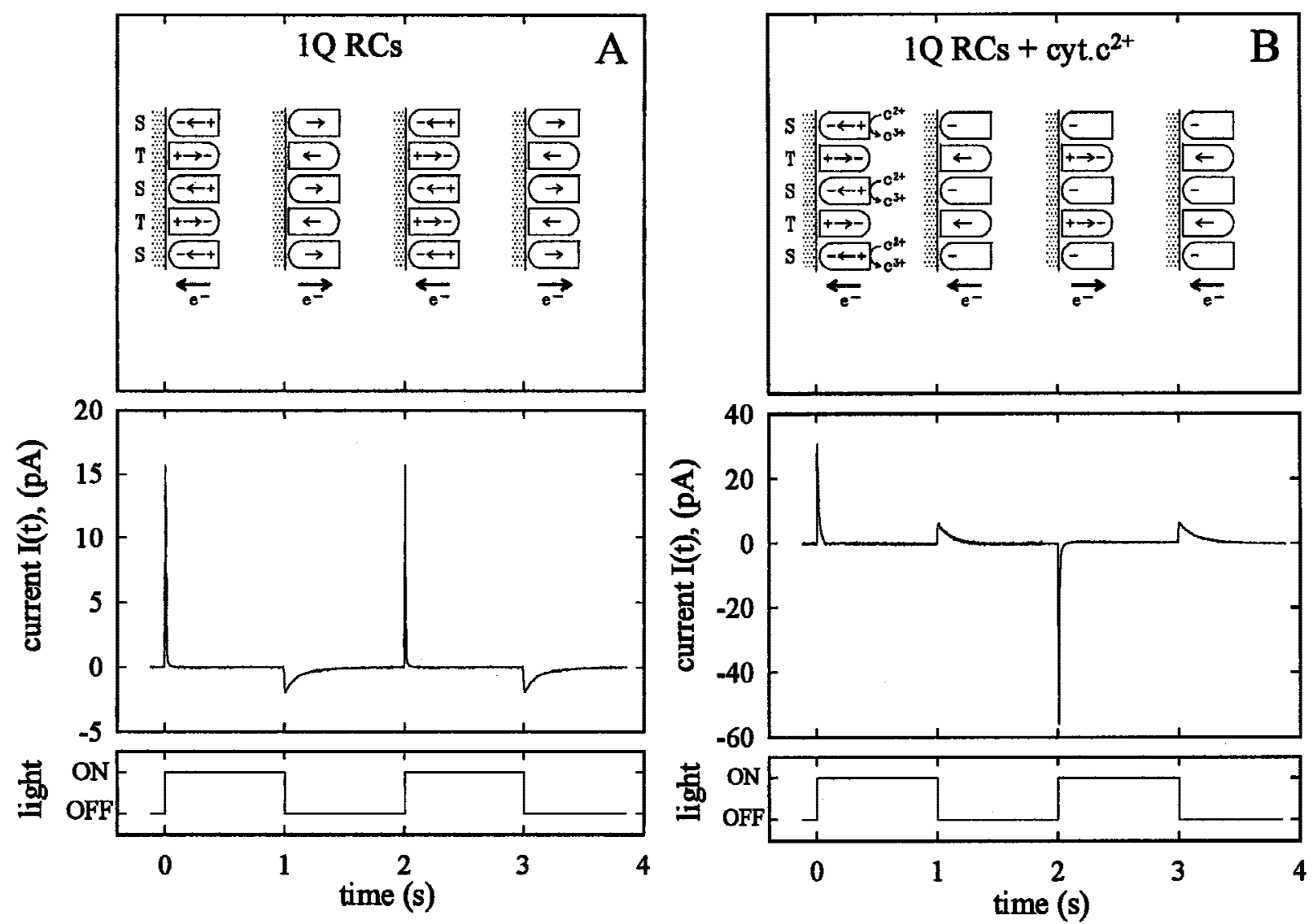

Fig. 7. The current, $I(t)$, in response to illumination of the $R C$-lipid interfacial layer. The upper drawings show the electronic state of both RC populations (denoted by $S$ and $T$, respectively). The dotted region symbolizes the Teflon film. The arrows indicate the direction of the electron transfer in those $R C$ s that are photochemically active and the bold arrows indicate the direction of the net electron transfer in the interfacial layer. The RCs in the layer contained $<5 \%$ functional $Q_{B}$. Thus the state $P^{+} Q_{A}^{-}$was formed upon illumination. The illumination sequence is shown at the bottom. The response time of the experimental setup was limited by the opening and closing times of the shutter $(\sim 1$ msec). Reaction conditions were the same as in Fig. 4. (A) The current response for two illumination periods in the absence of cytochrome $c^{2+}$. The peak amplitude of the charge recombination current is smaller than that of the charge separation but the area under all the curves is the same because the total charge transferred in both reactions is the same. Traces are averages of 16 experiments. $(B)$ Light-induced currents in the presence of $40 \mu \mathrm{M}$ cytochrome $c^{2+}$. During the first illumination, RCs in the $S$ population are inactivated when cytochrome $c^{2+}$ reduces $P^{+}$. During the second and subsequent illuminations only the $T$ population RCs are photochemically active. The right trace (second illumination) is an average of 16 experiments (note the smaller noise level than on the left trace (first illumination)). Note that the current scale is different than in $(A)$.

to a ratio of 2 in aqueous detergent solution (Okamura et al., 1982). This difference is not surprising in view of the hydrophobic nature of $U Q_{10}$ which partitions preferentially in the lipid of the interfacial layer but prefers the $R C$ in aqueous detergent solution.

\section{The Effective Orientation of RCs IN THE LAyeR}

The effective orientation of $R C \mathrm{~s}$ in the interfacial layer was determined by comparing the initial chargerecombination current generated by both $R C$ populations to that generated by just the $\mathrm{T}$ population. The transient current generated by one quinone $R C \mathrm{~s}$ in an interfacial layer following a series of light flashes in the absence of cytochrome $c^{2+}$ is shown in Fig. 7A. The current generated when the light is turned on is associated with charge separation and its time integral divided by $C_{T}$ equals the voltage rise shown in Fig. 4 (Eq. 3). The current when the light is turned off is associated with the $P^{+} Q_{A}^{-} \rightarrow P Q_{A}$ charge recombination and its time integral divided by $C_{T}$ equals the fast voltage decay shown in Fig. 4 (Eq. 3).

The light induced current generated by an interfacial layer containing one-quinone RCs in the presence of cytochrome $c^{2+}$ is shown in Fig. $7 B$ (note the different scale of the ordinate compared to Fig. 7A). Reduction of $P^{+}$by cytochrome $c^{2+}$ produced the inactive $P Q_{A}^{-}$state in the $S$ population (see top frame of Fig. $7 B$ ). Consequently, only the $T$ population recombined following illumination which generated a current of opposite sign to the recom- 
bination current in the absence of cytochrome $c^{2+}$ (Fig. $7 A$ ). Subsequent light pulses (one is shown in Fig. 7B) generated currents corresponding to charge separation and recombination in only the $T$ population.

The effective orientation of the RCs was calculated using Eq. 13 and the measured peak currents associated with the charge recombination shown in Fig. 7. The value of $\Theta$ at $\mathrm{pH} 8.0$ was determined to be:

$\Theta=0.14 \pm 0.03$.

$\Theta>0$ signifies there are more $R C$ s in the $S$ population than in the $T$ population. The error in $\Theta$ is due to variations of the orientation in different interfacial layer preparations (8 interfacial layers were used.).

\section{The Density of RCs IN the InTERfacial Layer}

The density of the $R C \mathrm{~s}$ in the layer was determined using Eq. 14. The integral of the measured current associated with charge recombination $P^{+} Q_{A}^{-} \rightarrow P Q_{A}$ (see Fig. 7A), obtained from measurements on 8 interfacial layers, was:

$\int_{0}^{t_{\max }} I_{A P}(t) d t=-0.22 \pm 0.06 \mathrm{pC}$

The distance between $Q_{A}$ and $P$ (center to center of the rings) projected onto the two fold symmetry axis, which is parallel to the normal of the interfacial layer plane, was determined from the $R C$ structure to be $\vec{d}_{A P} \cdot \hat{n} \cong 27$ $\AA$ (Allen et al., 1987; Yeates et al., 1987). Assuming the dielectric constant between $P$ and $Q_{A}, \varepsilon_{A P}$, to be equal to the average dielectric constant of the $R C$-lipid interfacial layer $\left(\varepsilon_{L}\right)$, and the average thickness of the layer around the $R C \mathrm{~s} D_{L}$ to be $\sim 40 \AA$ (see above and legend to Fig. 3), we find $\left|\alpha_{A P}\right| \cong 0.68$ (Eq. 5). If we further assume that the entire area of the Teflon film is covered by the lipid interfacial layer, i.e., $A_{L}=A_{T}$, the value of $\sigma$ obtained from Eq. 14 is:

$\sigma=(1.5 \pm 0.4) \cdot 10^{11} R C \mathrm{~s} / \mathrm{cm}^{2}$

This value is approximately equal to the density of $R C \mathrm{~s}$ found in chromatophores of $R$. sphaeroides (Packham et al., 1978). For an $R C$ with an elliptical cross section with minor and major radii of 20 and $30 \AA$ (Yeates et al., 1987 ) this density corresponds to a $\sim 2 \%$ coverage of the surface area of the interfacial layer.

\section{Conclusions}

We have characterized a simple and relatively robust system to measure electrogenic events in reaction centers incorporated into a phospholipid interfacial layer. The measured orientation of the $R C \mathrm{~s}$ in the interfacial layer was $\Theta=0.14 \pm 0.03$ at $\mathrm{pH} 8.0$, indicating that in $\sim 60 \%$ of the $R C$ s the surface nearest the donor was facing the aqueous solution. The density of $R C \mathrm{~s}$ in the interfacial layer was estimated to be $\sigma=(1.5 \pm 0.4) \cdot 10^{11} R C \mathrm{~s} /$ $\mathrm{cm}^{2}$. Nearly complete $(90 \%)$ binding of the secondary quinone $\left(Q_{B}\right)$ to the $R C$ was achieved by adding 100 $U Q_{10} / R C$ to the vesicle preparation.

An equivalent electrical circuit for the experimental system is presented and analyzed. The system can be used to investigate electrogenic processes that take place on a time scale shorter than $\sim 10 \mathrm{sec}$, which is the time constant of the electrical circuit corresponding to the leakage of ions through the interfacial layer. We are using the system to delineate various electron transfer steps, to study proton uptake in native and mutant $R C \mathrm{~s}$, to investigate structural changes in the $R C$, and to elucidate the dielectric properties of the protein.

We thank G. Feher, M. Montal, and M.Y. Okamura for their guidance, E.C. Abresch for preparing the RCs, R.A. Isaacson and G. Kassabian for helping with construction of the amplifiers, and M. Huber, N.M. Kroll, M.L. Paddock, P.H. McPherson, and S. Rongey for helpful discussions. Part of the work was done in the laboratory of G. Feher. The work was supported by a Swedish Natural Science Research Council fellowship to P.B., a National Institutes of Health Hemoglobin and Blood Protein Training Grant fellowship to A.M., and grants from the NSF and the National Institutes of Health to G. Feher.

\section{References}

Allen, J.P., Feher, G., Yeates, T.O., Komiya, H., Rees, D.C. 1987. Structure of the reaction center from Rhodobacter sphaeroides R26 I. The cofactors. Proc. Natl. Acad. Sci. USA 84:5730-734

Apell, H.-J., Snozzi, M., Bachofen, R. 1983. Kinetic analysis of bacterial reaction centers reconstituted in lipid bilayers. Biochim. Biophys. Acta 724:258-277

Brzezinski, P., Okamura, M.Y., Feher, G. 1992. Structural changes following the formation of $\mathrm{D}^{+} \mathrm{Q}_{A}^{-}$in bacterial reaction centers. In: The Photosynthesis Bacterial Reaction Center II: Structure, Spectroscopy and Dynamics. J. Breton and A. Vermeglio, editors. pp. 321-330. Plenum Press, NY

Brzezinski, P., Paddock, M.L., Okamura, M.Y., Feher, G. 1991. Electrogenicity associated with proton uptake by $Q_{B}^{2-}$ in bacterial RCs. Biophys. J. 59:143a (Abst.)

Brzezinski, P., Paddock, M.L., Okamura, M.Y., Feher, G. 1997. Lightinduced electrogenic events associated with proton uptake upon forming $Q_{B}^{-}$in bacterial wild-type and mutant reaction centers. Biochim. Biophys. Acta 1321:149-156

Crofts, A.R., Wraight, C.A. 1983. The electrochemical domain of photosynthesis. Biochim. Biophys. Acta 726:149-185

Diner, B.A., Schenck, C.C., DeVitry, C. 1984. Effect of inhibitors, redox state, isoprenoid chain-length on the affinity of ubiquinone for the secondary acceptor binding-site in the reaction centers of photosynthetic bacteria. Biochim. Biophys. Acta 766:9-20

Drachev, L.A., Frolov, V.N., Kaulen, A.D., Liberman, E.A., Ostroumov, S.A., Plakunova, V.G., Semenov, A.Y., Skulachev, V.P. 1976. Reconstitution of biological molecular generators of electric current. J. Biol. Chem. 251:7059-7065

Dracheva, S.M., Drachev, I.A., Konstantinov, A.A., Semenov, A.Y., Schulachev, V.P., Arutjunjan, A.M., Shuvalov, V.A., Zaberezh- 
maya, S.M. 1988. Electrogenic steps in the redox reactions catalyzed by photosynthetic reaction-center complex from Rhodopseudomonas viridis. Eur. J. Biochem. 171:253-264

Fahr, A., Läuger, P., Bamberg, E. 1981. Photocurrent kinetics of purple-membrane sheets bound to plantar bilayer membranes. $J$. Membrane Biol. 60:51-62

Feher, G., Allen, J.P., Okamura, M.Y., Rees, D.C. 1989. Primary processes in bacterial photosynthesis: Structure and function of reaction centers. Nature 339:111-116

Feher, G., Arno, T.R., Okamura, M.Y. 1988. The effect of an electric field on the charge recombination rate of $\mathrm{D}^{+} \mathrm{Q}_{\mathrm{A}}^{-} \rightarrow \mathrm{DQ}_{\mathrm{A}}$ in reaction centers from Rhodobacter sphaeroides R-26. In: The Photosynthetic Bacterial Reaction Center. J. Breton and A. Vermeglio, editors. pp. 271-287. Plenum Press, NY

Feher, G., Okamura, M.Y. 1978. Chemical composition, properties of reaction centers. In: The Photosynthetic Bacteria, R.K. Clayton and W.R. Sistrom, editors. pp. 349-386. Plenum Press, NY

Gopher, A., Blatt, Y., Schönfeld, M., Okamura, M.Y., Feher, G., Montal, M. 1985. The effects of an applied electric field on the rate of charge recombination in reaction centers incorporated in planar lipid bilayers. Biophys. J. 48:311-320

Hong, F.T. 1980. Displacement photocurrent in pigment-containing biomembranes artificial and natural systems. In: Bioelectrochemistry: Ions, Surfaces, and Membranes. M. Blank, editor. pp. 211238. Am. Chem. Soc., Advances in Chemistry Series 188, Washington, D.C.

Hong, F.T., Montal, M. 1979. Bacteriorhodopsin in model membranes: A new component of the displacement photocurrent in the microsecond time scale. Biophys. J. 25:465-472

Jackson, J.B., Dutton, P.L. 1973. The kinetic and redox potentionmetric resolution of the carotenoid shift in Rhodopseudomonas chromatophores: Their relationship to electric field alternations in electron transport and energy coupling. Biochim. Biophys. Acta 325:102113

Höök, F., Brzezinski, P. 1994. Flash-Induced Electrogenic Events Associated with Electron and Proton Transfer in Photosystem II Reaction Centres Incorporated in Phospholipid Interfacial Layers. Biophys. J. 66:2066-2072

Kagawa, Y., Racker, E. 1971. Partial resolution of the enzymes catalyzing oxidative phosphorylation. J. Biol. Chem. 246:5477-5487

Läuger, P. 1972. Carrier mediated ion transport. Science 178:24-30

Läuger, P. 1991. Electrogenic Ion Pumps. Sinauer Associates, Sunderland, MA

Läuger, P., Benz, R., Stark, G., Bamberg, E., Jordan, P.C., Fahr, A., Brock, W. 1981. Relaxation studies of ion transport systems in lipid bilayer membranes. Q. Rev. Biophys. 14:513-598

McPherson, P.M., Okamura, M.Y., Feher, G. 1990. Electron transfer from the reaction center of $R b$. sphaeroides to the quinone pool: Doubly reduced $\mathrm{Q}_{\mathrm{B}}$ leaves the reaction center. Biochim. Biophys. Acta 1016:289-292

Nelson, N., Anholt, R., Lindstrom, J., Montal, M. 1980. Reconstitution of purified acetylcholine receptors with functional ion channels in planar lipid bilayers. Proc. Natl. Acad. Sci. USA 77:3057-3061

Okajima, T.L., Hong, F.T. 1986. Kinetic analysis of displacement photocurrents elicited in two types of bacteriorhodopsin model membranes. Biophys. J. 50:901-912

Okamura, M.Y., Debus, R.J., Kleinfeld, D., Feher, G. 1982. Quinone binding sites in reaction centers from photosynthetic bacteria. In: Function of Quinones in Energy Conserving Systems. B.L. Trumpower, editor. pp. 299-317. Academic Press

Okamura, M.Y., Isaacson, R.A., Feher, G. 1975. The primary acceptor in bacterial photosynthesis: The obligatory role of ubiquinone in photoactive reaction centers of Rhodopseudomonas sphaeroides. Proc. Natl. Acad. Sci. USA 72:3491-3495
Packham, N.K., Berriman, J.A., Jackson, J.B. 1978. The charging capacitance of the chromatophore membrane. FEBS Lett. 89:205-210

Packham, N.K., Dutton, L.P., Mueller, P. 1982. Photoelectric currents across planar bilayer membranes containing bacterial reaction centers. Response under conditions of single electron turnover. Biophys. J. 37:465-473

Packham, N.K., Packham, C., Mueller, P., Tiede, D.M., Dutton, L.P. 1980. Reconstitution of photochemically active reaction centers in planar phospholipid membranes: Light induced currents under voltage clamped conditions. FEBS Lett. 110:101-106

Rayfield, G.W. 1982. Kinetics of the light-driven proton movement in model membranes containing bacteriorhodopsin. Biophys. J. 38:79-84

Rosen, P., Pecht, I. 1976. Conformational equilibria accompanying the electron transfer between cytochrome $c$ (P551) and azurin from Pseudomas aeruginosa. Biochem. 15:775-786

Schönfeld, M., Montal, M., Feher, G. 1979. Functional reconstitution of photosynthetic reaction centers in planar lipid bilayers. Proc. Natl. Acad. Sci. USA 76:6351-6355

Schönfeld, M., Montal, M., Feher, G. 1980. Reaction centerphospholipid complex in organic solvents: formation and properties. Biochemistry 19:1535-42

Tiede, D.M., Mueller, P., Dutton, L.P. 1982. Spectrophotometric and voltage clamp characterization of monolayers of bacterial photosynthetic reaction centers. Biochim. Biophys. Acta 681:191-201

Trissl, H.-W. 1981. The concept of chemical capacitance: A critique. Biophys. J. 33:233-242

Trissl, H.-W. 1983. Spatial correlation between primary redox components in reaction centers of Rhodopseudomonas sphaeroides measured by two electrical methods in the nanosecond range. Proc. Natl. Acad. Sci. USA 80:7173-7177

Trissl, H.-W., Darszon, A., Montal, M. 1977. Rhodopsin in model membranes: Charge displacements in interfacial layers. Proc. Natl. Acad. Sci. USA 74:201-210

Trissl, H.-W., Montal, M. 1977. Electrical demonstration of rapid lightinduced conformational changes in bacteriorhodopsin. Nature 266:655-657

Westman, H.P., 1956. editor. Reference Data for Radio Engineers, 4th edition. Stratford Press, NY

Witt, H.T. 1971. Coupling of quanta, electrons, fields, ions, and phosphorylation in the functional membrane of photosynthesis. Q. Rev. Biophys. 4:365-477

Wraight, C.A. 1981. Oxidation-reduction physical chemistry of the acceptor quinone complex in bacterial photosynthetic reaction centers: evidence for a new model of herbicide activity. Israel J. Chem. 21:348-354

Yeates, T.O., Komiya, H., Rees, D.C., Allen, J.J., Feher, G. 1987. Structure of the reaction center from Rhodobacter sphaeroides R26 III. Membrane-protein interactions. Proc. Natl. Acad. Sci. USA 84:6438-6442

\section{Appendix A}

\section{Analysis of the Equivalent Circuit}

The equivalent electrical circuit (Fig. $3 b$ ) was analyzed to determine the electrical time constants and the relations between the $R C$-generated current, $i(t)$, and the measured current $I(t)$ and voltage $V(t)$. From Kirchoff's law:

$i(t)=i_{1}(t)+i_{2}(t)+I(t)$ 
A

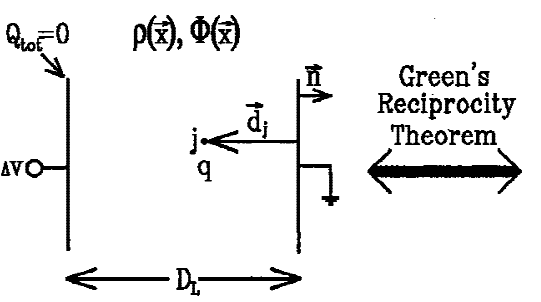

B

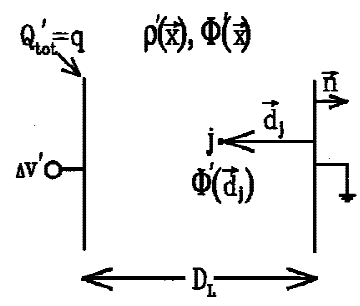

Fig. B1. Model to calculate the voltage generated across a lipid interfacial layer by a charge transfer within the interfacial layer (Appendix B). The interfacial layer is represented as a parallel-plate capacitor with one grounded and one ungrounded plate. $D_{L}$ is the interfacial layer thickness and $\hat{n}$ is a unit vector normal to the interfacial layer. The point $j$ is within the interfacial layer. $\vec{d}_{j}$ is a vector from the grounded conductor to $j$. (A) The charge distribution $\rho(\vec{x})$ consists of a charge $q$ in the $R C$ at the point $j$ and the charge induced on the interfacial layer surface. The ungrounded side of the interfacial layer is assumed to be neutral $\left(Q_{t o t}=0\right) . \rho(\vec{x})$ produces a potential $\Phi(\vec{x})$. On the ungrounded plate, $\Phi(\vec{x})$ is equal to the interfacial layer voltage, $\Delta v$. $(B)$ The complicated charge distribution $\rho(\vec{x})$ in part $A$ is related via Green's Reciprocity Theorem to the simple charge distribution $\rho^{\prime}(\vec{x})$, consisting of charge $q$ on the ungrounded plate $\left(Q_{t o t}^{\prime}=q\right)$ and the induced charge $(-q)$ on the grounded plate. $\rho^{\prime}(\vec{x})$ produces a potential $\Phi^{\prime}(\vec{x})$ with the value $\Phi^{\prime}\left(\vec{d}_{j}\right)$ at the point $j$.

Expressing $v(t)$ in terms of the currents through each leg of the circuit gives,

$v(t)=i_{1}(t) R_{L}=\frac{1}{C_{L}} \int_{o}^{t} i_{2}\left(t^{\prime}\right) d t^{\prime}=\frac{1}{C_{T}} \int_{o}^{t} I\left(t^{\prime}\right) d t^{\prime}+I(t) R_{e}$

where we have assumed that the monolayer and Teflon sheet capacitors are initially uncharged.

The solution of Eq. A1 and A2 for the case of an exponentially decaying $R C$-generated current, $i(t)=i_{O} e^{-t / \tau_{0}}$, where $\tau_{O}$ is the time constant of the associated electrogenic process, and the limit of $C_{L}>$ $C_{T}$, relevant to the present geometry, is:

$$
\begin{aligned}
I(t)= & -i_{0} \tau_{0} R_{L} C_{T}\left[\frac{e^{-t / \tau_{O}}}{\left(\tau_{o}-\tau_{1}\right)\left(\tau_{0}-\tau_{2}\right)}\right. \\
& \left.+\frac{e^{-t / \tau_{1}}}{\left(\tau_{1}-\tau_{0}\right)\left(\tau_{1}-\tau_{2}\right)}+\frac{e^{-t / \tau_{2}}}{\left(\tau_{2}-\tau_{0}\right)\left(\tau_{2}-\tau_{1}\right)}\right]
\end{aligned}
$$

where $\tau_{1}=\mathrm{R}_{L} \mathrm{C}_{L}$ and $\tau_{2}=\mathrm{R}_{e} \mathrm{C}_{T}$. Equation A3 states that the measured voltage $\left(V(t)=I(t) R_{e}\right)$ is in general triphasic, with the time constant of the electogenic reaction, $\tau_{O}$, and the two electrical time constants, $\tau_{1}$ and $\tau_{2}$, all contributing to the measured signal.

For $t$ and $\tau_{0}<<\tau_{1}$ and $\tau_{2}$, the measured voltage is

$V(t) \cong \frac{i_{0} \tau_{O}}{C_{L}}\left(1-e^{-t / \tau o}\right)$.

$V(t)$ increases with the kinetics of the charge transfer in the reaction center to a voltage equal to the total charge transferred $\left(\int_{O}^{\infty} i(t) d t=i_{O} \tau_{O}\right)$ divided by the capacitance of the layer. Substitution of $i(t)=i_{0} e^{-t / \tau_{0}}$ into Eq. 3 yields Eq. A4.

On the longer time scale, Eq. A3 shows that the voltage increase $\left(V(t)=I(t) R_{e}\right)$ will be followed by a decay with the electrical time constants $\tau_{1}$ and $\tau_{2}$. When one of these time constants is much smaller than the other, then, for times short compared to the longer time constant, the measured voltage decays essentially monophasically with the smaller time constant. These predictions are in agreement with measurements made during continuous illumination of the monolayer where the voltage was observed to rise with the kinetics of the opening time of the shutter $(\sim 1 \mathrm{msec})$ and to decay monophasically with the smaller of the two electrical times, $\tau_{1}$ or $\tau_{2}$ (Fig. 5).
For current measurements, $R_{e}=0$ and Eq. A3 simplifies to give the measured current,

$I(t)=\frac{-i_{O} \tau_{O} R_{L} C_{T}}{\tau_{O}-\tau_{1}}\left(\frac{e^{-t / \tau_{O}}}{\tau_{O}}-\frac{e^{-t / \tau_{I}}}{\tau_{1}}\right)$

which reflects the kinetics of both the charge transfer, $\tau_{0}$, and the electrical time constant, $\tau_{1}$. For $t$ and $\tau_{O} \ll<\tau_{1}$, Eq. A5 simplifies to Eq. 4 with $i(t)=i_{0} e^{-t / \tau 0}$.

\section{Appendix B}

\section{Voltage Generated by a Charge Transfer Within THE MONOLAYER}

To derive the voltage generated across the interfacial layer by $N$ dipoles in its interior (Eq. 7) we first consider the effect of a single charge in the interfacial layer. The interfacial layer was modeled as a parallel plate capacitor with one plate grounded (Fig. B1) and one plate ungrounded and neutral.

For any two charge distributions, $\rho(\vec{x})$ and $\rho^{\prime}(\vec{x})$, Green's Reciprocity Theorem states,

$\int \rho(\vec{x}) \Phi^{\prime}(\vec{x}) d^{3} \vec{x}=\int \rho^{\prime}(\vec{x}) \Phi(\vec{x}) d^{3} \vec{x}$

where the integrals are over all space. The first charge distribution $\rho(\vec{x})$ consists of a charge $q$ at a point $j$ between the capacitor plates and the induced charge distribution on the capacitor plates (Fig. B1A). The charge distribution $\rho(\vec{x})$ produces a potential $\Phi(\vec{x})$. The second charge distribution $\rho^{\prime}(\vec{x})$, consists of charge $q$ on the ungrounded capacitor plate and the charge $-q$ induced on the ungrounded plate. $\rho^{\prime}(\vec{x})$ produces the potential $\Phi^{\prime}(\vec{x})$ (Fig. B1B).

The integrals in Eq. B1 were broken into integrals over the ungrounded plate, the region between the plates, and the grounded plate. The integral over the grounded plates vanishes because the potential there is zero. The potentials on the ungrounded plates, $\Delta v$ and $\Delta v^{\prime}$, are constant and factor out of the integrals over these plates. The remaining integrals of the charge distribution over the ungrounded plates yield 
the total charge on these plates, $Q_{t o t}$ and $Q^{\prime}{ }_{t o t}$ respectively. Thus Eq. B1 reduces to,

$Q_{t o t} \Delta v^{\prime}+q \Phi^{\prime}\left(\vec{d}_{j}\right)=Q_{t o t}^{\prime} \Delta v$

where $\vec{d}_{j}$ is a vector from the grounded plate to point $j$. Substitution of $Q_{t o t}=0$ for the total charge on the neutral plate and $Q_{t o t}^{\prime}=q$ yields,

$\Delta v=\Phi^{\prime}\left(\vec{d}_{j}\right)$

which states that the voltage across a capacitor due to a charge $q$ located at point $j$ between the capacitor plates is equal to the potential at $j$ when the charge is on the ungrounded plate.

For a parallel plate capacitor, $\Phi^{\prime}\left(\vec{d}_{j}\right)$ is given by the negative line integral of the electric field from the grounded plate to the point $j$,

$\Phi^{\prime}\left(\vec{d}_{j}\right)=-\int_{o}^{j} \vec{E} \cdot d \vec{l}=\frac{q}{\varepsilon_{j} A_{L}} \vec{d}_{j} \cdot \hat{n}$

where $(\vec{E})$ is the electric field as determined from Gauss' Law, $\varepsilon_{j}$ is the dielectric constant at $j, A_{L}$ is the area of the interfacial layer, and $\hat{n}$ is a unit vector normal to the interfacial layer.
The voltage across the interfacial layer is due to dipoles that consist of charge $q_{j k}$ at point $j$ and $-q_{j k}$ at point $k$. These dipoles effectively form a parallel plate capacitor, and the voltage across the interfacial layer due to $N$ such dipoles is arrived at by superposition of the result in Eq. B4,

$\Delta v_{j k}(t)=\frac{N q_{j k}}{\varepsilon_{j k} A_{L}} \vec{d}_{j k} \cdot \hat{n}=\frac{N q_{j k}}{C_{L}}\left(\frac{\varepsilon_{L}}{\varepsilon_{j k}} \frac{\vec{d}_{j k} \cdot \hat{n}}{D_{L}}\right)$

where $\vec{d}_{j k}=\vec{d}_{j}-\vec{d}_{k}$ is the distance vector that separates the dipole charges and $\varepsilon_{j k}$ is the dielectric constant in a slab, containing all the dipoles, that is parallel to the plates of the interfacial layer. The first equality in Eq. B5 shows that the generated voltage does not depend on the thickness of the interfacial layer, the position of the dipoles, or the dielectric constant outside of the slab. The voltage is independent of these quantities because the entire voltage drop occurs across the slab containing the dipoles. The generated voltage is expressed, in the second equality of Eq. B5 (Eq. 7), in terms of the parallel plate capacitance of the interfacial layer, $C_{L}=\varepsilon_{L} A_{L} / D_{L}$, where $\varepsilon_{L}$ is the average dielectric constant of the interfacial layer and $D_{L}$ is the thickness of the layer. The term in parenthesis is Läuger's dielectric coefficient $\alpha_{j k}$ (Läuger, 1991). 